\title{
Relacionamiento de Instituciones de Educación Superior Colombianas con El Sector Empresarial: un Análisis Reflexivo
}

Herrera Cárdenas, Claudia ${ }^{1\left(^{*}\right)}$; Gutiérrez, José María ${ }^{2}$ \& Sanabria, Carolina ${ }^{3}$

${ }^{1}$ Corporación Universitaria de Sabaneta -Unisabaneta, Facultad de Negocios y Ciencias Empresariales, Sabaneta, Antioquia, Colombia.

${ }^{2}$ Tecnologico de Antioquia Institución Universitaria, Facultad de Ciencias Administrativas y Económicas, Medellín, Colombia. ${ }^{3}$ Alcaldía Municipal de Rionegro Antioquia, Secretaría de Educación, Rionegro Antioquia, Colombia.

\section{RESUMEN}

El papel que han venido jugando históricamente las instituciones de educación superior en el desarrollo de las naciones, ha sido fundamental, sin embargo, en países como Colombia, la conexión entre las universidades como centros de investigación y el sector empresarial, no ha sido tan fuerte, dado que las pequeñas y medianas empresas no han visto reflejadas en su gran mayoría, los beneficios de la investigación realizada en sus instalaciones. Este estudio de revisión, presenta un análisis de distintas publicaciones científicas adelantadas por varias universidades de la Ciudad de Medellín al sector empresarial. Se concluye que las universidades colombianas deben mejorar diametralmente sus vínculos y convenios con el sector empresarial, de modo que no se abarquen solo a algunas empresas por medio de proyectos, sino que se busque que las universidades puedan generar sus propios laboratorios de investigación social, técnica y científica en el seno de las empresas, teniendo como meta, darle cobertura a miles y miles de empresas que han carecido del acompañamiento y asesoría de las universidades para garantizar sus sostenibilidad y futuro.

Palabras clave: : Universidades; Empresas; Proyectos; Investigación; Revisión

\section{Relationship of Colombian Higher Education Institutions with the Business Sector: A Reflective Analysis}

\section{ABSTRACT}

The role that higher education institutions have historically been playing in the development of nations has been fundamental, however, in countries like Colombia, the connection between universities as research centers and the business sector has not been so strong, given that small and medium-sized companies have not seen, for the most part, the benefits of the research carried out in their facilities. This review study presents an analysis of different scientific publications advanced by various universities of the City of Medellín to the business sector. It is concluded that Colombian universities must diametrically improve their links and agreements with the business sector, so that they do not cover only some companies through projects, but rather that universities can generate their own social and technical research laboratories. and scientific within the companies, with the goal of providing coverage to thousands and thousands of companies that have lacked the support and advice of universities to guarantee their sustainability and future.

Keywords: Universities; Business; Projects; Research; Review 


\section{INTRODUCCIÓN}

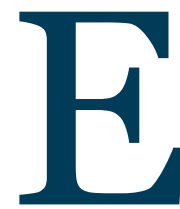

n este artículo de reflexión, se abordará la forma en que un conjunto de universidades públicas y privadas de la Ciudad de Medellín, Colombia, hacen sus nexos o conexiones con el sector empresarial local, más específicamente, en la forma en que estas aportan a las empresas, puesto que, la investigación y la innovación son el brazo armado de las instituciones de educación superior o por su sigla, más conocidas como IES, permitiendo que, con análisis documentales como este, sea posible adentrar al lector especializado y no especializado, en las dinámicas que tienen las universidades que operan dentro del territorio medellinense, en lo relativo a los modelos de negocios que tienen las empresas y a cómo las universidades se sintonizan con dichos modelos, discutiendo, tanto las ventajas como desventajas que poseen desde dichos modelos y a su vez, proponer un modelo que pueda ser de utilidad para las universidades investigadas (Navarrete, 2013).

La educación superior es uno de los sectores considerados en la frontera del conocimiento, pues, históricamente, es en las universidades en donde se gesta gran parte del conocimiento de la humanidad y es en ellas en donde se producen las más innovadoras metodologías de investigación, así como de educación. El énfasis que poseen las universidades se han centrado en primera medida en la educación formal, en segunda instancia han concentrado sus esfuerzos en investigación y en tercera posición se ubica una alta inversión en servicios de extensión, lo que hace que, en muchos casos, no se vea de forma tan nítida y clara, la relación establecida entre las universidades y el sector empresarial (Ibarra \& Villagrán, 2013). Este manuscrito tiene como objetivo, presentar dicha relación en diferentes apartados, que parten con la introducción, prosiguen con el marco teórico, la metodología, hasta culminar con la discusión y las conclusiones.

\section{MARCO TEÓRICO}

Para el caso de la República de Colombia, las empresas se dividen por tamaño, según la cantidad de personal que trabaje al interior de ellas de manera directa, razón por la cual, se habla de grandes, medianas y pequeñas empresas, siendo estas dos últimas, denominadas por la sigla de PYME, que se traduce como pequeñas y medianas empresas, haciendo que, sea uno de los sectores en que se observan mayores acercamientos por parte de grupos de Investigación, Desarrollo e innovación o $I+D+i$, que nacen al interior de las instituciones de educación superior colombianas y que denotan grandes esfuerzos por conectar sus progresos y avances hacia el sector empresarial (Bojalil, 2008). La educación superior contemporánea, se define como un conjunto de aprendizajes y capacitaciones de tipo terciario, que están organizados y segmentados mediante actividades institucionalizadas, las que incluyen en grado mayor a las universidades convencionales o tradicionales, en donde dicha educación terciaria, ha perseguido la meta de crear mallas curriculares en todas las áreas del conocimiento, pasando por artes, hasta llegar a las humanidades, y teniendo una gran fortaleza e inversión en las facultades de ciencias básicas e ingenierías, factor este, que le ha permitido a las instituciones universitarias, ir obteniendo cada vez más especializaciones, maestrías, doctorados y posdoctorados. El concepto propiamente dicho de educación superior también incluye a las instituciones postsecundarias como los politécnicos, los colegios de educación ingenieril y " las grandes école", que es un término francés, usado para designar a instituciones universitarias enfocadas en un área específica. Bajo este paraguas de la educación superior, es que se vienen a concebir todas las formas de instituciones profesionales, tanto en los países desarrollados como en los países en vías de desarrollo, incluso, este amplio espectro no agota las posibilidades de las formas de educación superior, pues, en el caso de Colombia, las instituciones de educación superior tienen corporaciones universitarias, fundaciones universitarias y universidades (Casanova, 2016). 
Una universidad, por otra parte, representa y simboliza dentro de las sociedades humanas, tanto a instituciones de educación superior en todo el sentido legal de la expresión, así como a toda una comunidad de académicos y científicos, que son los modernos eruditos de todas las naciones, haciendo que, se los tenga como la clase social privilegiada en materia intelectual. Una universidad es una institución dedicada a la educación superior, la cual tiene como meta e ideal, formar a hombres y mujeres en los más altos niveles y estándares de desarrollo intelectual, tanto en las artes, como en las humanidades y las ciencias, haciendo que, por medio de los grupos de investigación, congresos y revistas científicas, puedan llevar siempre a sus respectivas disciplinas al siguiente escalón del conocimiento. Una universidad es una fuente de universalidad, de ahí su nombre, por lo que, los conocimientos que producen día a día, les confiere tener uno de los poderes humanos más altamente reconocidos: la generación de nuevo conocimiento, pues las personas formadas y entrenadas en las aulas universitarias durante tantos años, hacen de ellas, seres humanos muy calificados dentro de sus respectivas profesiones, elevando de forma simultánea, la calidad de vida de las naciones que más inversión hacen en formación integral (Sebastián, 2005).

Las instituciones de educación superior también se dedican a la formación de la clase media técnica y tecnóloga, por lo que, la fuerza de la educación terciaria, extiende sus brazos hacía todas las direcciones en las cuales, el sector empresarial requiera mano de obra, bien sea especializada en el grado más elevado posible o en labores de manufactura mecánica que no requieran de un grado muy alto de cualificación, más si de destreza, lo que hace que las universidades sean matrices desde donde las grandes ciudades o centros urbanos pueden mejorar sus prácticas laborales, ya que de ellas surge el personal más calificado para las tareas que cada época demande. Es necesario elucidar que, las universidades, así como los idiomas, las culturas y los pueblos, tienen diferencias, pues pese a que ofrezcan carreras similares, todas ellas tienen su propia identidad, identidad esta, que se refleja en la formación que imprimen en el personal estudiantil y profesional que se forma en sus aulas, por lo que, no encontraremos universidades $u$ otras instituciones de educación superior con la misma identidad, ya que tienden a diferir en su misión, en sus objetivos, en sus funciones, en la forma de calificación requerida por cada facultad, en los criterios de admisión para los estudiantes, en la duración de los programas que ofertan y en el tipo de certificación que otorgan a los egresados (Fuenmayor, 2003).

El término universidad, viene siendo acuñado en la Europa Medieval, para la designación de centros dedicados a la enseñanza de lo que, desde una postura más teológica se conoce como ciencias sagradas, en donde se impartían asignaturas enfocadas en la formación monacal, clerical y también de las clases oligarcas y por supuesto, de la realeza, lo que, con el paso de los siglos, fue generando una mayor apertura y fundación de muchas más universidades alrededor de Europa y con la llegada de las Coronas Inglesa, Española y Portuguesa a las Américas, también comenzaron a proliferar por todas las actuales naciones de Norteamérica, Centroamérica y Suramérica. Es solo en los últimos doscientos años que, las universidades comenzaron a ofrecer carreras orientadas a las ciencias exactas y naturales, pero siempre conservando su impronta de fe en todos sus cuerpos estudiantiles. Esta expansión de los centros universitarios por todo el mundo, se fue despojando paulatinamente de la formación religiosa, para enfocarse más en la gestación de nuevas carreras, nuevas disciplinas científicas, nuevos laboratorios, nuevas bibliotecas abiertas a la población en general y una enorme apertura a personas de todas las clases sociales, lo cual, ha sido pieza clave para hacer que las universidades sean centros de enseñanza de elite y en su mayor parte, que estén en sintonía con Estados laicos, lo que es una variable en favor del pensamiento crítico y objetivo (Tommasino \& Cano, 2016).

Las universidades han ido prosperando a lo largo del último siglo, se han ido multiplicando y es tanto el éxito que ha tenido este tipo de sistemas educa- 
tivos que, a la fecha se cuentan más de 25,000 instituciones de educación superior en los cinco Continentes, lo que significa que, las universidades se han ido convirtiendo en un referente de obtención de conocimiento, de clase intelectual, de poder político y social y claro está, de poder científico y tecnológico. En la actualidad, oír nombrar universidades como Harvard University, el Massachusetts Institute of Technology - MIT, Stanford University, Caltech, Princeton University o cualquier universidad que sea referente dentro de cualquier país del mundo, se asocia automáticamente a la excelencia académica y a la clase intelectual más virtuosa y más cualificada, razón por la cual, las universidades son estandartes de la calidad educativa llevada a su máxima expresión, lo que no excluye a Latinoamérica, en donde se pueden denotar famosas universidades como la Universidad Autónoma de México - UNAM, la Universidad de Buenos Aires - UBA, la Universidad Pontificia Católica de Chile, la Universidad Nacional de Colombia, entre muchas otras, pues no importa si se habla de universidades de naciones del primer mundo o de universidades de países tercermundistas, ya que es en el corazón de las universidades, en donde se gestan los mayores logros científicos, tecnológicos y de innovación que una nación pueda tener y ello es materia de estudio por derecho propio (Chomsky, 2017).

\section{METODOLOGÍA}

La metodología empleada para este artículo de reflexión, consistió en la revisión documental de la información relativa a conexiones entre universidades públicas y universidades privadas ubicadas dentro del casco urbano de la Ciudad de Medellín. La búsqueda consistió en recuperar datos propios de las páginas web de ocho importantes universidades de la mencionada urbe y posteriormente, analizar sus modelos de trabajo mancomunado para o con el sector empresarial, además de proponer un modelo propio en el que se puedan ofrecer sugerencias $y$ recomendaciones a las universidades en cuestión. Dichas instituciones de educación superior se enumeran del siguiente modo, comenzando por las universidades de carácter público: 1) Universidad de
Antioquia - UdeA; 2) Instituto Tecnológico Metropolitano - ITM; 3) Politécnico Colombiano Jaime Isaza Cadavid y 4) Tecnológico de Antioquia - Institución Universitaria, TdeA. Por otro lado, están las cuatro universidades privadas de mayor reconocimiento y renombre, las cuales se enumeran del siguiente modo: a) Universidad Pontificia Bolivariana - UPB; b) Universidad de Medellín - UDEM; c) Universidad EAFIT y d) Universidad CES.

En el caso de la Universidad de Antioquia - UdeA, se han revisado a autores como Zuluaga \& Rodríguez (2018), pues dan cuenta de la vinculación que dicha institución de educación superior sostiene con el sector empresarial farmacéutico y biotecnológico, lo que es clara evidencia de la relación que sostiene esta universidad con esas dos áreas de negocios. En tanto que, el Instituto Tecnológico Metropolitano ITM, por medio de autores como Baena, Zuleta \& Calderón (2018), hace enormes contribuciones al sector empresarial para el desarrollo de materiales y biocombustibles, lo que la hace ser una institución vanguardista en ese terreno. Por su parte, el Politécnico Colombiano Jaime Isaza Cadavid, demuestra amplia experiencia en el sector agropecuario por medio de autores como Gómez, Londoño \& Madrid (2013), quienes dan cuenta de la relevancia que como institución de educación superior tienen al vincularse con el sector lechero, que es uno de los más fuertes y sólidos en el Departamento de Antioquia. En cuanto al Tecnológico de Antioquia - Institución Universitaria, TdeA, autores como Gil (2019), dan cuenta de la interrelación exitosa que en recientes años ha venido sosteniendo como universidad junto al sector contable, financiero y económico de la Ciudad de Medellín, haciendo aportes muy significativos en pro del desarrollo equitativo del sector empresarial local.

En lo que concierne a las instituciones de educación superior de carácter privado, se comienza enunciando los aportes hechos por autores como TrujiIlo-Agudelo, Ramírez-Quintero \& Arango-Barrientos (2018), quienes desde la Universidad Pontificia Bolivariana están demostrando que esta institución se encuentra generando vínculos sumamente sólidos con el sector empresarial de la salud, lo que 
es un indicador muy positivo de las iniciativas de universidades privadas hacia el sector médico en atención de problemas de salud. En cuanto a la Universidad de Medellín - UDEM, autores de talla mundial como lo son Quiceno \& Vinaccia (2019), autoridades en ciencias de la salud y calidad de vida y que han abierto las puertas de esta universidad al abordaje del sector privado hospitalario, clínico y de atención especializada en enfermedades crónicas, están generando nuevas propuestas, sistemas de evaluación y medición para la calidad de vida y la salud en los colombianos. Mientras tanto, la Universidad EAFIT, está sumando importantes esfuerzos por vincular sus grupos de investigación en ciencias de la nutrición, proteómica y ciencias médicas para fortalecer la industria farmacéutica local, tal y como lo confirman autores como O'Donovan \& Cadena-Gaitán (2018), quienes, desde el seno de dicha universidad, fortalecen a la misma con avances para el control de la diabetes y el cuidado medioambiental. Para cerrar este apartado de instituciones de educación superior privadas, se abordan los aportes de la Universidad CES, la cual, por medio de autores como Sav, Rotondo, Syro, Serna \& Kovacs (2019), está permitiendo que la universidad se vincule estrechamente al sector empresarial inmunológico, virológico y de desarrollo de tecnologías biomédicas, lo que históricamente, ha venido siendo uno de los pilares de esta institución.

Las ocho instituciones de educación superior que han sido seleccionadas, lo fueron con base en cinco criterios que son: 1) Tener el estatus de universidad acreditada por el Ministerio de Educación Nacional MEN de la República de Colombia; 2) Contar con grupos de investigación en las más altas clasificaciones del Ministerio de Ciencia, Tecnología e Innovación - Minciencias de Colombia; 3) Poseer publicaciones científicas en las más relevantes Bases de Datos como Scopus, Web of Science, ESCl o PubMed; 4) Destacarse por su alta competitividad y 5) Ser reconocidas en los más importantes rankings nacionales e internacionales debido a su calidad académica, formativa, científica y productiva. Los puntos anteriormente enunciados, ponen de manifiesto que, todas ellas son instituciones sumamente presti- giosas dentro de la Ciudad de Medellín, llegándose a posicionar como referentes de talla mundial en diferentes sectores empresariales.

\section{RESULTADOS Y/O DISCUSIÓ}

La postura de que las universidades deben conectarse con mucha mayor eficiencia y calidad con el sector empresarial, es una tesis defendida en este manuscrito, debido a que, cuando el sector empresarial más pudiente y el sector empresarial emergente, optan de forma decidida y con absoluta determinación por hacer fuertes inversiones en investigaciones llevadas a cabo en el seno de las universidades, se hace mucho más sencillo dar el salto de ciencias básicas a ciencias aplicadas, razón por la cual, se respalda el referente teórico de Zurmehly \& Adams (2017), quienes postulan que, las universidades deben estar profundamente vinculadas al sector empresarial, para que se generen soluciones sociales, en materia de salud, en materia de educación y en todos los sectores, dándole especial preponderancia al desarrollo de nuevas tecnologías educativas y formativas, y claro está, por expandir con la mayor eficiencia posible, las tecnologías de la información y la comunicación para hacer que la educación sea accesible a poblaciones y zonas geográficas sumamente distanciadas de los grandes cascos urbanos, de modo que la educación recibida en las zonas rurales sea de la misma calidad que la recibida en zonas urbanas.

Como contraparte, se encuentran autores como Patel \& Cespedes (2016), quienes sostienen que es más prioritario enfocarse en lo que pueden gestionar las grandes empresas, mega-corporaciones y multinacionales para llevar servicios educativos y formativos a todo el mundo, lo que difiere de la postura defendida en este manuscrito, dado que, históricamente, las universidades han demostrado ser faros de conocimiento para la humanidad y no debería desconectarse la educación que proveen las universidades, de las propuestas e intereses de las grandes compañías, puesto que, tanto el sector empresarial como el sector de la educación terciaria o superior, tienen elementos indispensables para el crecimiento, desarrollo y evolución de las empresas. 
Esto quiere decir que, tendría que existir no solo una comunicación fluida y constante entre el sector universitario y el sector empresarial, sino que, deberían existir Decretos de Ley en donde las empresas más grandes, al igual que las medianas y pequeñas empresas, puedan vincularse a la resolución de los problemas o dificultades empresariales a partir de la mayor ventaja que poseen las universidades: la investigación. Este ejercicio tendría que surgir a partir del Estado y debería consolidarse año tras año, de modo tal que, resulte indiferenciable el sector empresarial del sector universitario, pues ambos estarían apuntando a los mismos objetivos.

Por tanto, el modelo que se sugiere en este manuscrito, es que las instituciones de educación superior estudiadas, puedan conducir sus esfuerzos educativos a las empresas más nuevas, artesanales y que hayan sido creadas para el sostenimiento de pequeñas comunidades, pues llevando las metodologías e innovaciones educativas que poseen las universidades, es que resultaría factible pensar en un verdadero fortalecimiento del pequeño empresario. Esto es lo que se recomienda como modelo, debido a que no se encontraron publicaciones o innovaciones empresariales en donde se apoye a empresas generadas bajo preceptos de equidad de género, empresas de origen étnico, empresas de personas en situación de discapacidad y empresas familiares que busquen el sostenimiento y la sustentabilidad de sus comunidades, por lo que ahí es en donde se recomienda que estas ocho universidades deben entrar a liderar proyectos que no sean aislados u ocasionales para los sectores mencionados, sino que estén en capacidad de dar cobertura para todos ellos.

\section{CONCLUSIONES}

Este estudio ha encontrado que, tras revisar en importantes Bases de Datos las publicaciones científicas de ocho reconocidas universidades medellinenses, existe una amplia relación entre las susodichas y el sector empresarial, lo que probablemente se deba a que los egresados de las mismas, deben salir a integrarse como fuerza laboral en los diferentes sectores empresariales que ofrece la Ciudad de
Medellín, razón por la cual, es una consecuencia lógica que dichas universidades con tanta tradición y reconocimiento, enfoquen sus programas profesionales, sus especializaciones, sus maestrías y sus doctorados en alinearse con las compañías productoras de bienes y servicios.

Las oportunidades que estas ocho instituciones de educación superior podrían encontrar para mejorar, sería creando alianzas permanentes para, desde sus experiencias con grandes corporaciones, llegar a fortalecer a las pequeñas y medianas empresas no solo de Medellín, sino de todo el Departamento de Antioquia, pues esos esfuerzos y esa experiencia, le podrá imprimir el sello de innovación que poseen dichas universidades a las empresas que año tras año nacen en la ciudad, permitiendo con ello, evitar que gran parte de esas microempresas se vayan a la quiebra por su inexperiencia, escasos o nulos conocimientos en investigación empresarial y claro está, esta podría constituirse como una plataforma para que las universidades no se enmarquen solo en lo académico o en sus vínculos con grandes corporaciones, sino que estarían en condiciones de ayudar a todas esas empresas incipientes que requieren el apoyo y el respaldo de instituciones con tanta experticia como las ya analizadas.

Finalmente, el sector universitario colombiano debe tener una apuesta no solo desde la creación o generación de documentos legales en los que se firmen convenios con otras instituciones de educación superior del Continente o del resto del mundo, sino que debe abrirse a exportar sus investigadores e incluso, grupos enteros de investigación, no para experiencias como pasantías o posdoctorados, sino para permitir que nuestros científicos lleven las aportaciones y desarrollos locales a sectores empresariales de las naciones más lejanas y con más altos niveles de calidad de vida, para que se les permita a dichas empresas, contar con la experticia de los investigadores colombianos y que estos a su vez, puedan aprender cómo extrapolar las ventajas y fortalezas de cientos o miles de casos de éxito empresarial, para hacerlos realidad en el contexto colombiano, pues dicha apuesta, sería el primer paso para salir del subdesarrollo en pocas décadas. 


\section{REFERENCIAS}

Baena, L., Zuleta, E., \& Calderón, J. (2018). Evaluation of the Stability of Polymeric Materials Exposed to Palm Biodiesel and Biodiesel-Organic Acid Blends. Polymers, 10(5). pii: E511. doi: 10.3390/polym10050511

Bojalil, L. (2008). La relación universidad-sociedad y sus desafíos actuales. REencuentro. Análisis de Problemas Universitarios, 52, 11-18.

Casanova, H. (2016). Modelos universitarios emergentes. Universidades, 67,4-5.

Chomsky, N. (2017). El asalto neoliberal a las universidades y cómo debería ser la educación. Tareas, 155, 35-48.

Fuenmayor, L. (2003). Diferencias entre universidades privadas y oficiales venezolanas: Algunos ajustes necesarios en el sector privado. Revista Venezolana de Gerencia, 8(22), 245-269.

Gil, J. (2019). Contabilidad Social, Desarrollo Equitativo y Universidad Critica: Interrelaciones y Dependencias. En-Contexto Revista de Investigación en Administración, Contabilidad, Economía y Sociedad, 7(10), 53-67.

Gómez, J., Londoño, L, \& Madrid, V. (2013). El perfil metabólico como herramienta de monitoreo de la salud, la producción y la fertilidad en el hato lechero del Politécnico Colombiano Jaime Isaza Cadavid. Revista Lasallista de Investigación, 10(1), 38-48.

Ibarra, J., \& Villagrán, A. (2013). La investigación en las universidades privadas: ¿un lujo o una necesidad? Universidades, 55, 48-55.

Navarrete, Z. (2013). La universidad como espacio de Formación profesional y constructora de identidades. Universidades, 57, 5-16.

O'Donovan, G., \& Cadena-Gaitán, C. (2020). Air pollution and diabetes: it's time to get active! The Lancet. Planetary Health, 2(7), e287-e288. doi: 10.1016/S2542-5196(18)30148-7

Patel, B. N., \& Cespedes, F. V. (2016). Introduction to Business Strategy. Journal of the American College of Radiology, 13(6), 747-749. doi:10.1016/j.jacr.2015.11.019

Quiceno, J., \& Vinaccia, S. (2019). Percepción de enfermedad y características sociodemográficas en enfermos crónicos. Revista Psicología y Salud, 28(2), 159-166.
Sav, A., Rotondo, F., Syro, L., Serna, C., \& Kovacs, K. (2019). Pituitary pathology in traumatic brain injury: a review. Pituitary, 22(3), 201-211. doi: 10.1007/s11102-019-00958-8

Sebastián, J. (2005). La internacionalización de las universidades como estrategia para el desarrollo institucional. Innovación Educativa, 5(26), 1-11.

Tommasino, H., \& Cano, A. (2016). Modelos de extensión universitaria en las universidades latinoamericanas en el siglo XXI: tendencias y controversias. Universidades, 67, 7-24.

Trujillo-Agudelo, D., Ramirez-Quintero, J., \& Arango-Barrientos, M. (2018). Successful treatment of heparin-induced thrombocytopenia with apixaban in a patient with chronic kidney disease requiring hemodialysis. Clinical Case Reports, 6(11), 2147-2149. doi: 10.1002/ccr3.1817

Zuluaga, A.F., \& Rodríguez, C. A. (2018). La ruta abreviada de comparabilidad colombiana para medicamentos biotecnológicos: ¿tendencia global o caso único? Revista Panamericana de Salud Pública, 41, e94.

Zurmehly, J., \& Adams, K. (2017). Using Quick Response Codes in the Classroom. CIN: Computers, Informatics, Nursing, 35(10), 505-511. doi:10.1097/cin.0000000000000363 